\title{
Upside-down Drawing in Posterior Cortical Atrophy
}

\author{
Marina Boban, Marina Mioč
}

Keywords: Posterior cortical atrophy, Rey-Osterrieth Complex figure

A 66-year-old man presented to the outpatient clinic of the Department of Cognitive Neurology, University Hospital Centre Zagreb with a 3-year history of progressive problems with reading, solving crossword puzzles, filling out bank slips, reaching objects in front of him and visual disorientation. At the time of presentation family reported that "he seemed like he could not see". His past medical history was positive for hypertension and chronic obstructive pulmonary disease, while family history was unremarkable. On the repeated ophthalmologic examination ocular problems were ruled out. In the neurocognitive examination, complete Balint's syndrome with oculomotor apraxia, simultanagnosia (inability to perceive more than one detail of visual stimulus and to integrate it into a coherent image), nonfoveal optic ataxia, and dyslexia with complete Gerstman's syndrome (dyscalculia, dysgraphia and fingers agnosia) were revealed. Additionally, significant visuospatial and visuoperceptual impairments were found. His copy of Rey-Osterrieth Complex figure (ROCF, Figure 1a) showed superimposition and upside-down reversal of perceived elements (Figure 1b). Episodic memory and executive functions were relatively spared compared to the deficits of higher order visual functions. His Mini-Mental State Examination score was 19/30. ${ }^{1}$ General neurological examination was unremarkable. Findings of laboratory tests (complete blood count, renal and thyroid function tests, vitamin $\mathrm{B}_{12}$, folic acid, immunology screening, serum and CSF antibodies to syphilis, Borrelia burgdorferi, tumor markers, hepatitis B and $\mathrm{C}$, human immunodeficiency virus (HIV), paraneoplastic antibodies), chest x-ray, electrocardiogram, electroencephalogram and visual evoked potentials were unremarkable except for mild hypercholesterolemia. Brain MRI (Figure 2) revealed bilateral parieto-occipital cortical atrophy without significant hippocampal atrophy or vascular disease.

Characteristic clinical symptoms (primarily progressive higher order visual dysfunction with relatively spared other cognitive functions) and scan findings (Figure 2) lead to the diagnosis of posterior cortical atrophy (PCA). Distortion of drawing is commonly seen in patients with PCA. ${ }^{2-4}$ Superimposition of elements of ROCF in this patient is probably caused by simultanagnosia, ${ }^{2-6}$ whereas an upside-down copy of a figure (not reported previously in patients with PCA) might be caused by impairment of visuo-vestibular processing. Despite upside-down copy of a figure our patient did not report $180^{\circ}$ room tilt illusion which has, so far, been reported in only one patient with PCA. ${ }^{5}$

\section{Statement of Authorship}

Concept: Boban. Acquisition of data: Boban. Analysis and interpretation of data: Boban and Mioč. Drafting of the manuscript: Boban and Mioč. Critical revision of the manuscript for

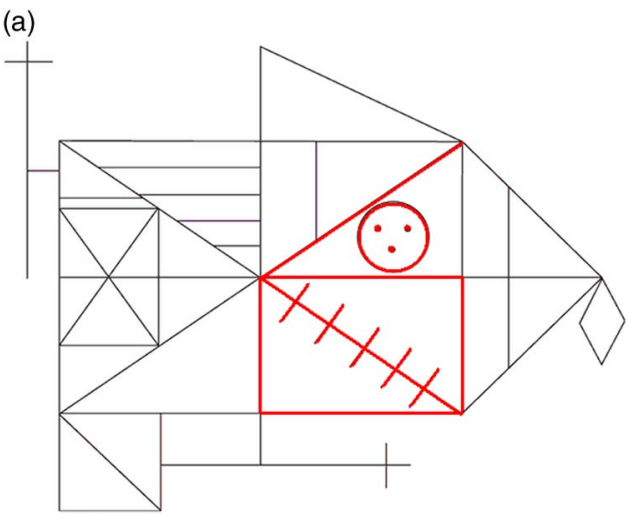

(b)

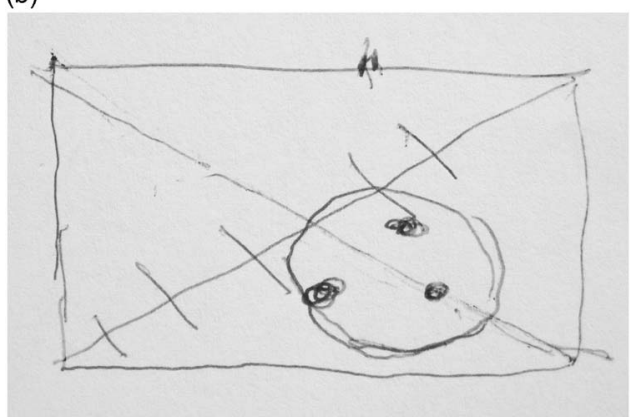

Figure 1: Rey-Osterrieth Complex figure and the patient's copy of figure. (a) Rey-Osterrieth Complex figure. Elements that were copied by the patient were marked in red color. (b) The patient's enlarged copy with superimposition of elements. Due to simultanagnosia patient was unable to perceive figure as a whole, but rather to notice only small fragments of figure. Upside-down reversal of copied elements is evident.

From the Department of Cognitive Neurology (MB), University Hospital Centre, Zagreb, Croatia and School of Medicine, University of Zagreb, Zagreb, Croatia; University Psychiatric Hospital Vrapče (MM), Zagreb, Croatia.

Received November 19, 2013. Final Revisions Submitted March 20, 2014. Correspondence to: Marina Boban, University Department of Cognitive Neurology, Zagreb School of Medicine and University Hospital Centre, Kišpatićeva 12; HR-10000 Zagreb, Croatia. Email: maboban@mef.hr 
(a)

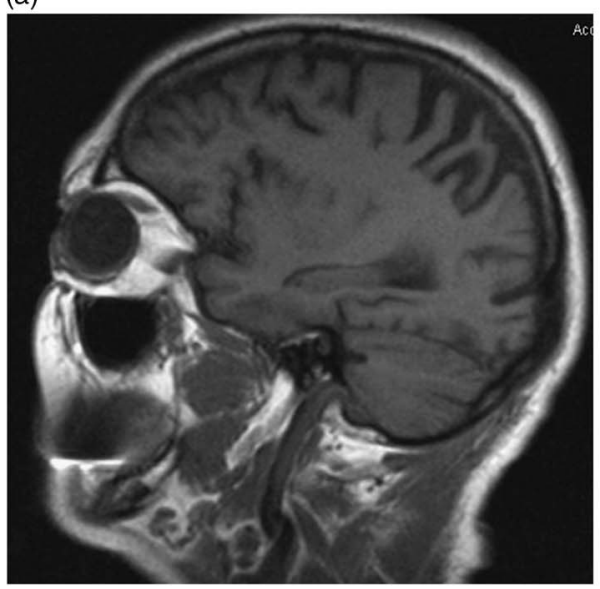

(b)

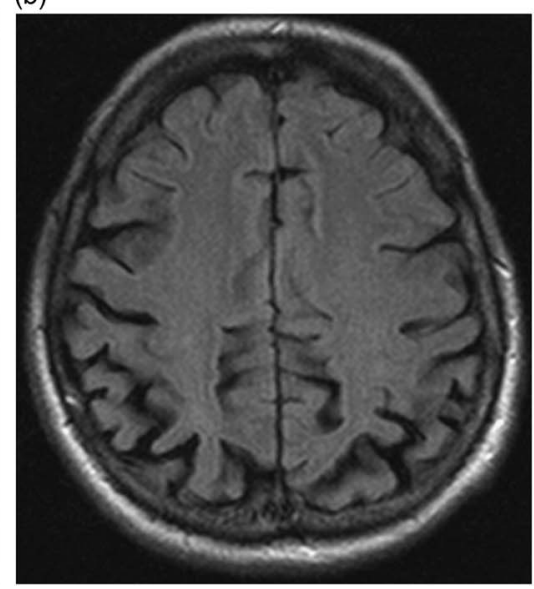

(c)

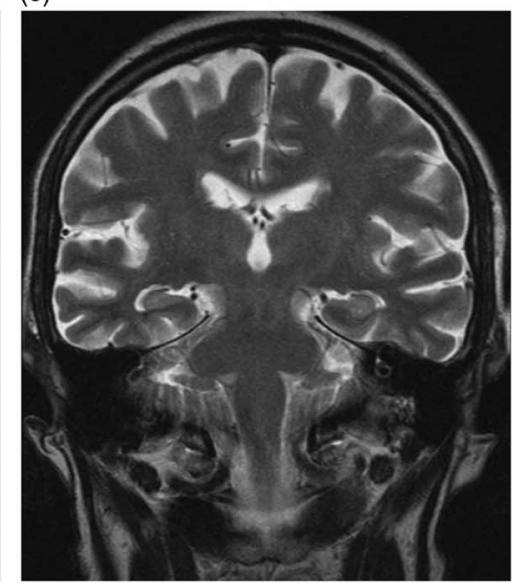

Figure 2: Brain MRI scan of a patient showing significant posterior cortical atrophy. (a) Sagittal view. (b) Axial view. (c) Coronal view showing relative sparing of hippocampal region.

important intellectual content: Boban and Mioč. Administrative, technical, and material support: Boban and Mioč.

\section{REFERENCES}

1. Boban M, Malojčić B, Mimica N, et al. The reliability and validity of the mini-mental state examination in the elderly Croatian population. Dement Geriatr Cogn Disord. 2012;33:385-92.

2. Crutch SJ, Lehmann M, Schott JM, Rabinovici GD, Rossor MN, Fox NC. Posterior cortical atrophy. Lancet Neurol. 2012;11:170-8.
3. Benson DF, Davis RJ, Snyder BD. Posterior cortical atrophy. Arch Neurol. 1988;45:789-93.

4. Iizuka O, Soma Y, Otsuki M, Endo K, Tanno Y, Tsuji S. Posterior cortical atrophy with incomplete Balint's syndrome. No To Shinkei. 1997;49:841-5.

5. Crutch SJ, Lehmann M, Gorgoraptis N, et al. Abnormal visual phenomena in posterior cortical atrophy. Neurocase. 2011;17:160-77.

6. McMonagle P, Kertesz A. Exploded drawing in posterior cortical atrophy. Neurology. 2006;67:1866. 